\title{
无机电致变色材料反射特性研究进展
}

\author{
张 翔 $^{1}$, 李文杰 ${ }^{2}$, 王乐滨 ${ }^{1}$, 陈 䀻 ${ }^{1}$, 赵九蓬 ${ }^{2}$, 李 圭 ${ }^{1}$ \\ (哈尔滨工业大学 1. 复合材料与结构研究所; 2. 化工与化学学院, 哈尔滨 150001)
}

摘 要: 电致变色材料是一种在外加电场下, 颜色可以发生可逆转变的材料。电致变色材料可调的光谱范围广, 可 以实现从可见到中远红外的宽波段调控, 在智能窗、显示、防炫目后视镜、智能热控和伪装等领域具有广泛应用前 景。目前对无机电致变色材料的研究大多是关于透过特性的研究, 对于反射特性的研究较少, 这主要是因为无机变 色材料大多颜色单一, 且不如有机变色材料容易设计。近年来, 通过一些特殊的制备方法和结构设计, 无机变色材 料反射特性的研究逐渐受到科研人员的重视。本文从无机电致变色材料的反射特性出发, 详细介绍了无机电致变色 在可见-近红外到中远红外波段的反射光谱调控方法和原理, 综述了最新研究进展。在可见波段, 反射特性调控主 要通过制备五氧化二钒及其掺杂化合物、一维光子晶体微结构、法布里-珀罗纳米谐振腔结构和局域表面等离子共 振等方式实现。在红外波段, 主要利用氧化铇等材料的分子振动吸收和德鲁德自由电子气体理论等理论设计制备红 外反射型电致变色器件。最后, 对未来无机电致变色材料反射调节的实际应用进行了展望。

关 键 词: 电致变色; 反射; 发射率; 无机材料; 多色; 综述

中图分类号: TQ174 文献标志码: A

\section{Reflective Property of Inorganic Electrochromic Materials}

\author{
ZHANG Xiang ${ }^{1}$, LI Wenjie ${ }^{2}$, WANG Lebin ${ }^{1}$, CHEN Xi $^{1}$, ZHAO Jiupeng $^{2}$, LI Yao ${ }^{1}$
}

(1. Center for Composite Materials and Structure, Harbin Institute of Technology, Harbin 150001, China; 2. School of Chemistry and Chemical Engineering, Harbin Institute of Technology, Harbin 150001, China)

\begin{abstract}
Optical property, such as color, transmittance, reflectance and emissivity, of electrochromic materials can be changed reversibly under low applied voltages. Electrochromic materials have a wide range of regulatable spectrum, which can realize the broadband control from the visible to mid-far-infrared. Electrochromic materials show a wide application prospect in the fields of intelligent window, display, anti-glare rearview mirror, intelligent thermal control, and camouflage. At present, most of researches on inorganic electrochromic materials focus mainly on transmission characteristics, but less on reflection characteristics. This is mainly because most inorganic electrochromic materials have single color and are not as easy to design as organic electrochromic materials. In recent years, through special preparation and structural design, the research on reflective properties of inorganic electrochromic materials has gradually attracted researchers' attention. Based on reflection characteristics of inorganic electrochromic materials, methods and principles of regulating the reflectance spectrum in the visible near
\end{abstract}

收稿日期: 2020-08-14; 收到修改稿日期：2020-09-22; 网络出版日期：2020-11-05

基金项目: 国家自然科学基金(52002097)

National Natural Science Foundation of China (52002097)

作者简介: 张 翔(1986-), 男, 讲师. E-mail: zhangxhit@hit.edu.cn

ZHANG Xiang(1986-), male, lecturer. E-mail: zhangxhit@hit.edu.cn

通信作者: 李 圭, 教授. E-mail: yaoli@hit.edu.cn

LI Yao, professor. E-mail: yaoli@hit.edu.cn 
infrared to mid-far-infrared bands are introduced, and the latest research progress is summarized. Within the visible band, reflectance spectrum control is mainly achieved by vanadium pentoxide $\left(\mathrm{V}_{2} \mathrm{O}_{5}\right)$ and $\mathrm{V}_{2} \mathrm{O}_{5}$ doping, microstructure of one dimensional photonic crystal, Fabry Perot nanocavity structure and localized surface plasmon resonance (LSPR). Within the mid-to-far infrared band, electrochromic devices (ECDs) based on the molecular vibration absorption of tungsten oxide $\left(\mathrm{WO}_{3}\right)$ or other electrochromic materials and related theory are designed and fabricated to regulate reflectance spectra. Finally, the practical application of inorganic electrochromic materials in future is prospected.

Key words: electrochromism; reflectance; emittance; inorganic material; multicolor; review

电致变色是指材料的光学特性, 包括透过率、 反射率、吸收率在外加电场下可逆改变的现象。1969 年, $\mathrm{Deb}^{[1]}$ 首先演示了以氧化铇 $\left(\mathrm{WO}_{3}\right)$ 为变色层的电 致变色器件。经过五十余年的发展, 对无机电致变 色材料的研究已较为成熟, 其中, 全无机薄膜材料 构筑的全固态电致变色器件是近年的研究热点, 并 且是较有应用潜力的研究方向 ${ }^{[2-4]}$ 。目前, 国内外 SAGE Electrochromics Inc. ${ }^{[5]}$ 、View Inc. ${ }^{[6]}$ 和浙江上 方电子装备有限公司 ${ }^{[7]}$ 等已具备一定的电致变色研 发生产能力。由于无机电致变色材料在变色过程中 的颜色属性较为单一, 目前对无机电致变色材料与 器件的研究主要集中于透过特性。例如, 常见的阴 极电致变色材料 $\mathrm{WO}_{3}$, 着色状态为蓝黑色, 褪色状 态为透明态 ${ }^{[8]}$; 阳极电致变色材料氧化镍 $(\mathrm{NiO})$, 着 色状态为灰褐色, 褪色状态为透明态 ${ }^{[9]}$ 。使用 $\mathrm{WO}_{3}$ 和 $\mathrm{NiO}$ 组装为器件时, 整体呈现黑色到透明的颜色 调节，因此具有很大的透过率调节范围 ${ }^{[10-11]}$ 。

实际上, 对于电致变色材料, 反射特性的调控 同样重要。在不同的波段范围, 反射特性调节的意义 也有所不同。在可见波段，对电致变色材料反射特性 的调节主要是调控材料的颜色，使其应用于伪装、显 示等领域 ${ }^{[2-13]}$ 。在中远红外波段, 可以对电致变色的 辐射特性进行调节, 即调控材料的发射率, 使其应 用于红外隐身、航天器智能热控等领域 ${ }^{[14-15]}$ 。有机 电致变色材料本身的特性使其更容易实现分子和结 构设计, 因此关于其电致变色反射特性调节的研究 更为广泛。但多数有机材料存在结构不稳定、紫外 辐照条件下易分解失效等问题, 使其在应用中受到 限制。而无机材料变色时, 颜色调控能力较单一, 只 有五氧化二钒 $\left(\mathrm{V}_{2} \mathrm{O}_{5}\right)$ 可以实现黄色到绿色的颜色转 变, 具有性能稳定的特点, 通过特殊的结构设计、掺 杂等也能实现特定的反射特性调控。本文详细介绍 了无机电致变色材料在可见-近红外和中远红外波 段反射特性的调控方法与性能, 并展望了未来无机 电致变色材料的反射调节的发展方向。

\section{1 无机电致变色材料在可见波段的反 射特性研究}

电致变色材料在可见波段的颜色变化具有重要 意义。由于电致变色材料具有双稳态的特性, 因此 用于静态显示具有很好的应用前景。同时, 部分电 致变色材料拥有典型的黄色到绿色的颜色转变, 在 伪装材料上也具有一定应用前景。无机电致变色多 色彩颜色转变通常通过 $\mathrm{V}_{2} \mathrm{O}_{5}$ 掺杂材料和构筑微结 构来实现。

\section{1 基于 $\mathrm{V}_{2} \mathrm{O}_{5}$ 的电致变色材料}

$\mathrm{V}_{2} \mathrm{O}_{5}$ 是一种研究广泛的过渡金属氧化物电致 变色材料, 为正交晶系材料, 晶体结构呈现层状结 构。在 $x y$ 平面上, 相邻的 $\mathrm{VO}_{6}$ 八面体共线相连接, 在 $z$ 轴方向上, 通过 $\mathrm{VO}_{6}$ 八面体定点之间共点实现 弱 $\mathrm{V}-\mathrm{O}$ 键连接。小体积离子在 $z$ 轴方向上嵌入/脱 出, 表现出电致变色性能 ${ }^{[16]} 。 \mathrm{~V}_{2} \mathrm{O}_{5}$ 在离子脱出状态 下表现为黄色, 在离子注入的条件下, 颜色逐步转 变为绿色或者蓝色以及中间状态的颜色, 从而可以 表现出多种颜色的变化, 反应方程式如下所示:

$$
\mathrm{V}_{2} \mathrm{O}_{5}+x \mathrm{Li}^{+}+x \mathrm{e}^{-} \rightleftharpoons \mathrm{Li}_{x} \mathrm{~V}_{2} \mathrm{O}_{5}
$$

Tong 等 ${ }^{[17]}$ 使用电沉积后热处理的方法, 制备 了珊瑚型 $\mathrm{V}_{2} \mathrm{O}_{5}$ 纳米棒结构, 该结构的 $\mathrm{V}_{2} \mathrm{O}_{5}$ 表现出 多色电致变色性能 (黄色、蓝绿色和橄榄色), 并具 有较好的稳定性, 如图 1(a,b)所示。Zhao 等 ${ }^{[18]}$ 采用 水热法和电沉积法相结合制备了表面粗粘的 $\mathrm{SnO}_{2} / \mathrm{V}_{2} \mathrm{O}_{5}$ 核壳复合薄膜, 核/壳结构对提高电致变 色性能起着关键性的作用, 由于反应面积大, 与基 底结合力强, 该复合膜具有优异的电致变色性能, 包括高调制对比度、高着色效率和良好的光学循环 稳定性。在电致变色薄膜中可观察到黄色、绿色和 蓝色之间可逆的颜色变化, 可以模拟沙漠、绿洲和 海洋环境下的颜色, 如图 1(c, d) 所示。Zhang 等 ${ }^{[19]}$ 以 $\mathrm{V}_{2} \mathrm{O}_{5}$ 和 $\mathrm{WO}_{3}$ 作为互补的电致变色层, 制备了 
glass $/ \mathrm{ITO} / \mathrm{WO}_{3} / \mathrm{Ta}_{2} \mathrm{O}_{5} / \mathrm{Li} / \mathrm{V}_{2} \mathrm{O}_{5} / \mathrm{ITO}$ 结构的全固态电 致变色器件, 该器件在着色时, 离子嵌入 $\mathrm{WO}_{3}$ 中, 显示颜色为 $\mathrm{WO}_{3}$ 蓝色和 $\mathrm{V}_{2} \mathrm{O}_{5}$ 黄色叠加得到的翠绿 色; 褪色时, 离子嵌入 $\mathrm{V}_{2} \mathrm{O}_{5}$ 中, $\mathrm{WO}_{3}$ 为透明无色, 器件显示为 $\mathrm{V}_{2} \mathrm{O}_{5}$ 的黄绿色。该颜色变化可以较好地 模拟沙漠、森林或者草地等场景的颜色, 如图 1(e,f) 所示。Zhang 等 ${ }^{[20]}$ 制备了基于锌钠钒氧化物 (Zn-SVO) 的电致变色显示器件, 该器件将锌夹在两
个 SVO 电极之间, 可以保持高光学透明度, 通过两 层 $\mathrm{V}_{2} \mathrm{O}_{5}$ 薄膜在不同电压下的颜色复配, 可在多种 颜色(橙色、琥珀色、黄色、棕色、浅绿色和绿色) 之间可逆切换，如图 1(g,h)所示。

\section{2 基于微纳结构的电致变色材料}

常见无机变色材料在可见波段颜色单一, 采用 $\mathrm{V}_{2} \mathrm{O}_{5}$ 和颜色复配可以实现的颜色转变较为有限。而 借鉴光子晶体概念，则可实现更丰富的颜色变化。

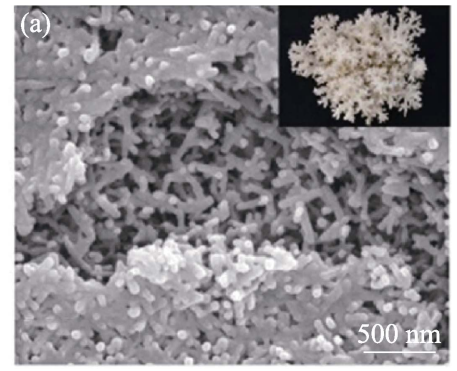

(b)
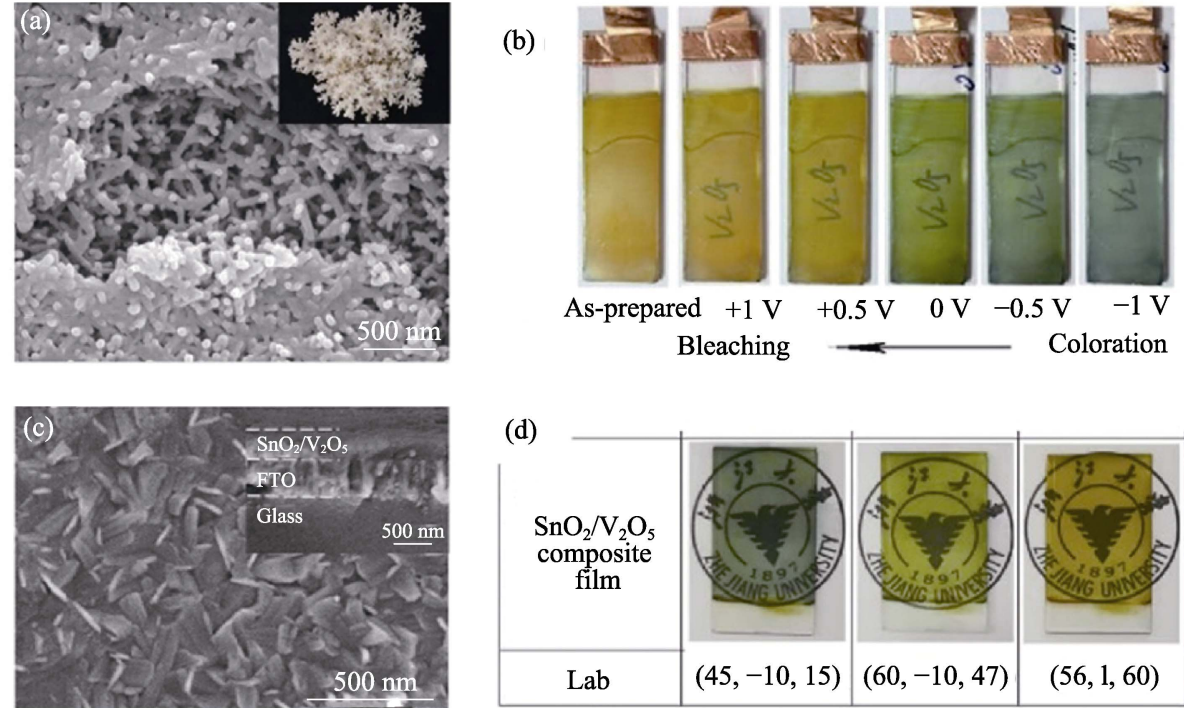

(d)
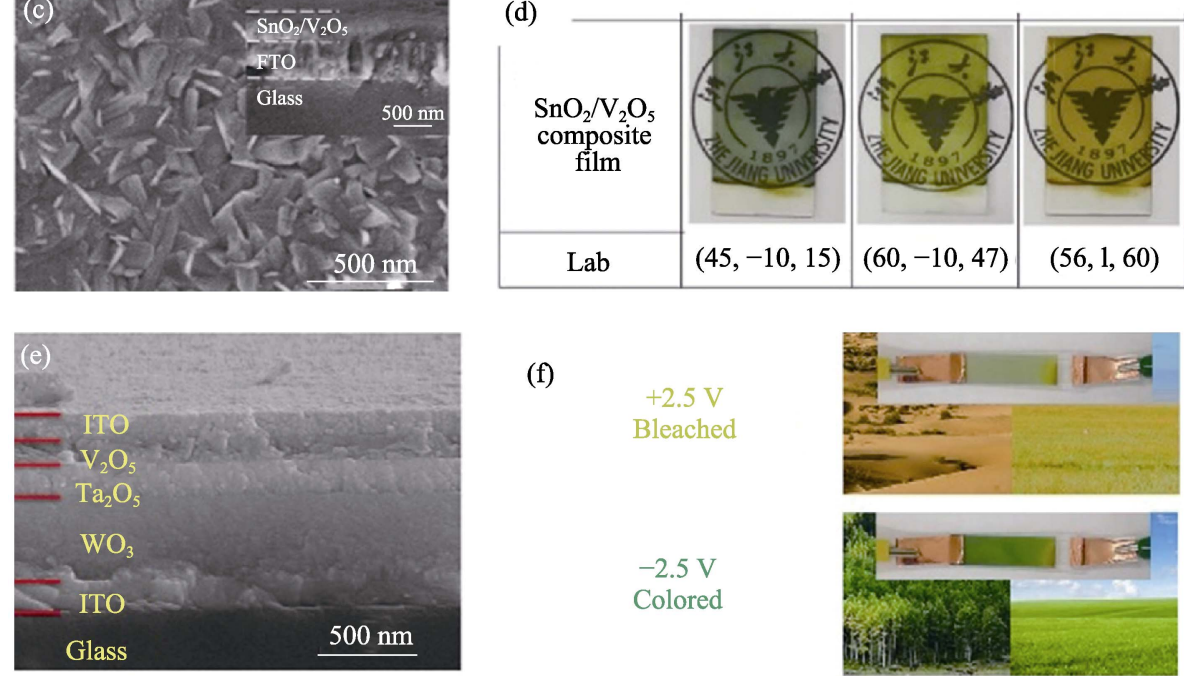

(f)
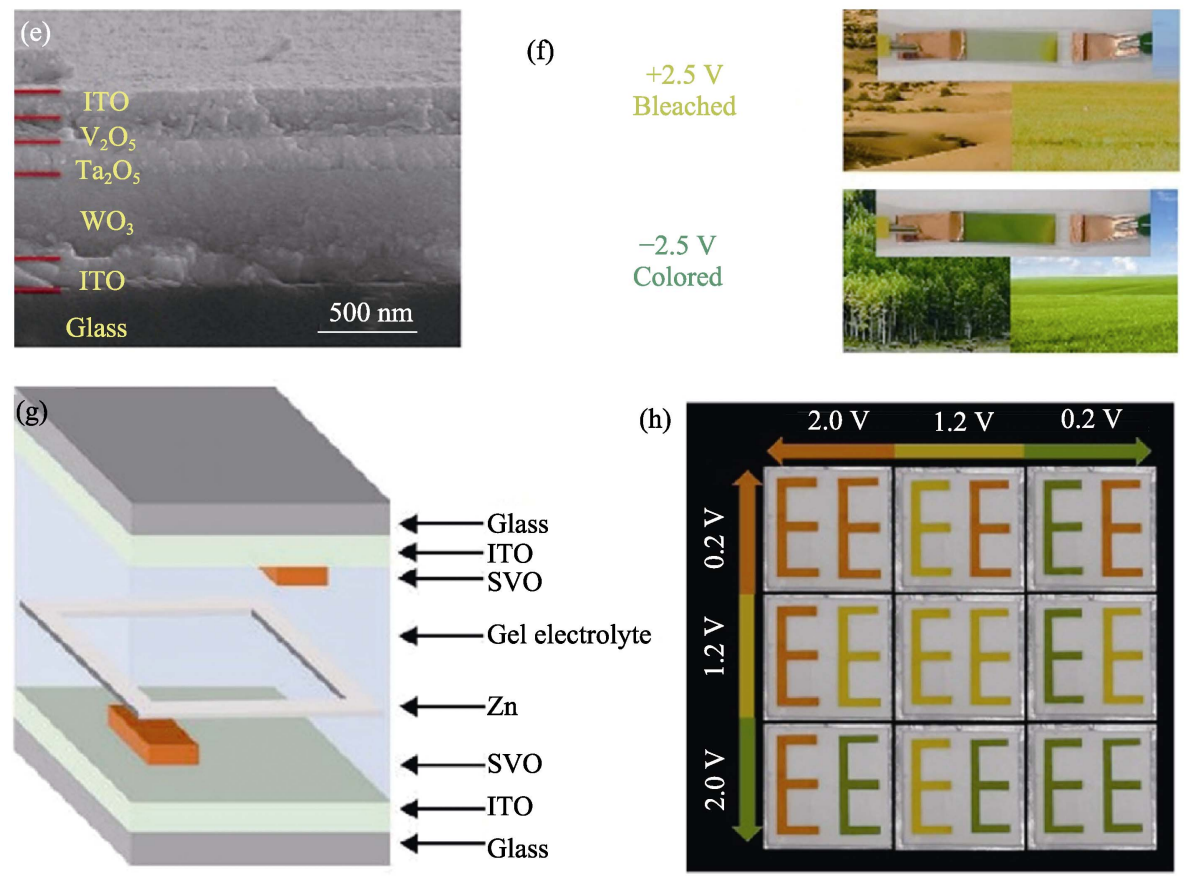

图 1 珊瑚状 $\mathrm{V}_{2} \mathrm{O}_{5}$ 纳米棒结构 SEM 照片(a), 珊瑚状 $\mathrm{V}_{2} \mathrm{O}_{5}$ 薄膜颜色对比(b), $\mathrm{SnO}_{2} / \mathrm{V}_{2} \mathrm{O}_{5}$ 薄膜的 SEM 照片 (c), $\mathrm{SnO}_{2} / \mathrm{V}_{2} \mathrm{O}_{5}$ 薄膜在不 同工作状态下的颜色参数(Lab 颜色模式)和光学照片(d), ITO/ $\mathrm{WO}_{3} / \mathrm{Ta}_{2} \mathrm{O}_{5} / \mathrm{Li} / \mathrm{V}_{2} \mathrm{O}_{5} / \mathrm{ITO}$ 器件的横截面 SEM 照片(e), 褪色和着色状 态下的器件实物照片(f), Zn-SVO 电致变色显示器件示意图 $(\mathrm{g})$, 以及 Zn-SVO 显示器件在不同电压下的数码照片(h) ${ }^{[17-20]}$

Fig. 1 Top-view SEM images of coralline $\mathrm{V}_{2} \mathrm{O}_{5}$ nanorod architecture (a), digital photos of coralline $\mathrm{V}_{2} \mathrm{O}_{5}$ architecture under different voltages (b), SEM images of $\mathrm{SnO}_{2} / \mathrm{V}_{2} \mathrm{O}_{5}$ films (c), color parameters (Lab color mode) and optical images of $\mathrm{SnO}_{2} / \mathrm{V}_{2} \mathrm{O}_{5}$ films at different working states (d), cross-sectional SEM image of the ITO/ $\mathrm{WO}_{3} / \mathrm{Ta}_{2} \mathrm{O}_{5} / \mathrm{Li} / \mathrm{V}_{2} \mathrm{O}_{5} / \mathrm{ITO}$ ECD (Electrochromic Device) (e), digital photos of the ECD in the bleached and colored state (f), schematic illustration of a large-scale Zn-SVO electrochromic display showing three intrinsic colors $(\mathrm{g})$, and digital photographs of the large-scale Zn-SVO display under different voltage bias conditions (h) ${ }^{[17-20]}$ 
光子晶体是指具有光子带隙特性的人造周期性电介 质结构, 其特点是具有不同折射率的材料周期排 列 ${ }^{[21]}$ 。Redel 等 ${ }^{[22]}$ 首先设计了基于纳米多孔的 $\mathrm{WO}_{3}$ 和 $\mathrm{NiO}$ 两种电致变色材料一维光子晶体结构, 多孔 结构保证了电解质离子在较厚的变色层中可以较好 地嵌入与脱出。首先, 当施加负偏压时, $\mathrm{WO}_{3}$ 着色, 折射率降低, $\mathrm{NiO}$ 褪色, 折射率升高, 导致两种材料 折射率对比下降。在构成一维光子晶体结构时, 由 于中心波长 $650 \mathrm{~nm}$ 有效折射率轻微地减小, 禁带轻 微地蓝移, 施加正向偏压, 折射率增大, 光子禁带 反射恢复。在此过程中, $\mathrm{WO}_{3}$ 和 $\mathrm{NiO}$ 叠层结构整体 呈现出从绿色、粉色到黄色的可逆变化, 如图 2(a) 所示。Xiao 等 ${ }^{[23]}$ 采用掠射角电子束蒸发技术, 在 ITO 基底上, 分别以 $75^{\circ}$ 和 $15^{\circ}$ 的掠射角沉积 $\mathrm{WO}_{3}$ 薄膜。由于不同掠射角沉积的 $\mathrm{WO}_{3}$ 薄膜具有明显不 同的微观结构和孔隙率，因此有效折射率也有明显 区别，两者折射率差值达到 0.41 。基于此，构成了以

(a)

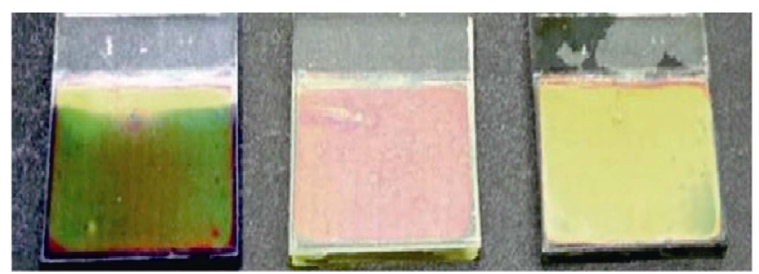

(b)

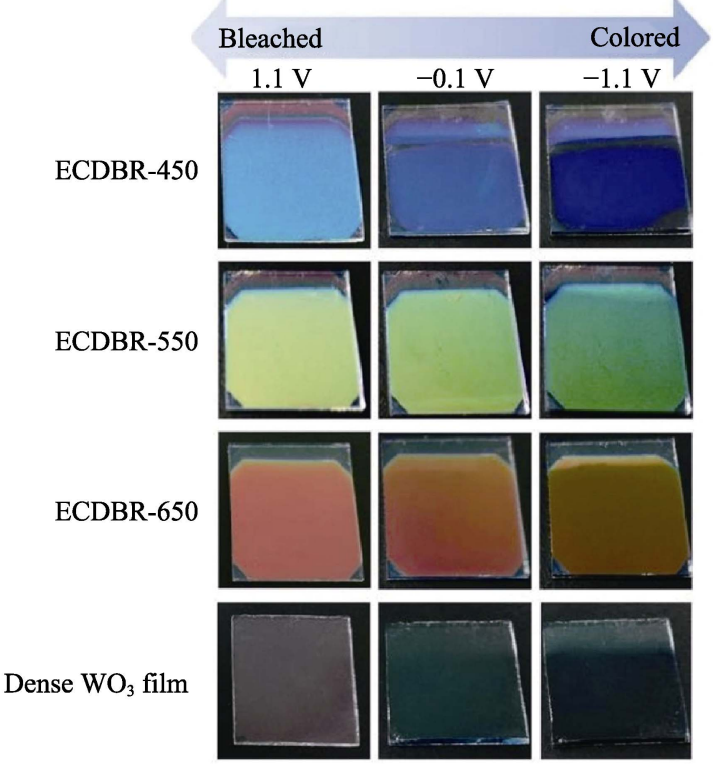

图 2 以 $60^{\circ}$ 角记录的具有 7 层周期结构的薄膜(橘红色)在阴 极着色 (黄色)和阳极着色(绿色)下的实物照片(a), 不同 Bragg 波长的致密 $\mathrm{WO}_{3}$ 薄膜和 4 周期双层电致变色分布 Bragg 反射器的电致变色特性(b) $\left(\lambda_{\mathrm{B}}=450,550,650 \mathrm{~nm}\right)^{[22-23]}$ Fig. 2 Color effects recorded at a $60^{\circ}$ angle for cathodic coloration (yellow stack) and anodic coloration (green stack) of an orange-red 7 double-layer nanoparticle $\mathrm{NiO} / \mathrm{WO}_{3}$ stack (a), and electrochromic properties of dense $\mathrm{WO}_{3}$ film and 4-bilayer electrochromic distributed Bragg reflectors with various Bragg wavelength (b) $\left(\lambda_{\mathrm{B}}=450,550,650 \mathrm{~nm}\right)^{[22-23]}$
不同折射率 $\mathrm{WO}_{3}$ 为周期结构的一维光子晶体。通过 设计不同的周期结构, 使这种光子晶体结构的中心 反射波长分别位于 450、550 和 $650 \mathrm{~nm}$ 。在电致变 色变色过程中, 反射中心波长移动最大可以达到 $72 \mathrm{~nm}$, 呈现出不同的颜色, 如图 2(b)所示。

除一维光子晶体结构外, 通过制备一种超紧凑 非对称的法布里-珀罗纳米谐振腔结构的电致变色 薄膜也可以实现丰富的颜色转变 ${ }^{[24]}$ 。该结构采用 $\mathrm{WO}_{3}$ 作为电致变色层, 金属 $\mathrm{W}$ 层同时作为电致变色 电极中的反射层和电流收集器。此时, 反射光谱中 会出现不同的共振, 光在 $\mathrm{WO}_{3}$ 薄膜的上下表面来回 反射，可以增强或抑制光的透射/反射，从而产生各 种结构颜色, 最大峰谷起伏高达 $56 \%$, 因此一些波 长的光被选择性地反射，而其他波长的光则通过光 干涉被吸收。随着 $\mathrm{WO}_{3}$ 层厚度的变化, 整体颜色会 呈现依赖于厚度的规律变化, 与电致变色相结合, 可以进一步丰富特殊的结构色，如图 3 所示。

利用局域表面等离子共振 (Localized Surface Plasmon Resonance, LSPR)也可以实现颜色的可逆 调控，即当光线入射到由贵金属(如 $\mathrm{Au} 、 \mathrm{Ag}$ 等)构成 的纳米颗粒上时，如果入射光子频率与贵金属纳米 颗粒或金属岛传导电子的整体振动频率相匹配时, 纳米颗粒或金属岛会对光子能量产生很强的吸收作 用。Kobayashi 等 ${ }^{[25-26]}$ 通过控制不同的电压和时间, 在 ITO 基底上沉积不同粒径与形状的 $\mathrm{Ag}$ 纳米粒子, 利用 $\mathrm{Ag}$ 纳米粒子的 LSPR, 实现了透明、银镜、红、 蓝和黑色五种光学状态的可逆调控, 如图 4(a)所示。 $\mathrm{Li}$ 等 ${ }^{[27]}$ 利用空心 $\mathrm{Au} / \mathrm{Ag}$ 合金纳米结构作为定位沉 积的稳定晶种, 消除了沉积过程中 $\mathrm{Ag}$ 随机自成核 现象, 优化了颜色转变的可逆性, 获得了多个可变 的对比明显的颜色，如图 4(b)。

综上所述, 采用无机材料制备的多色电致变色

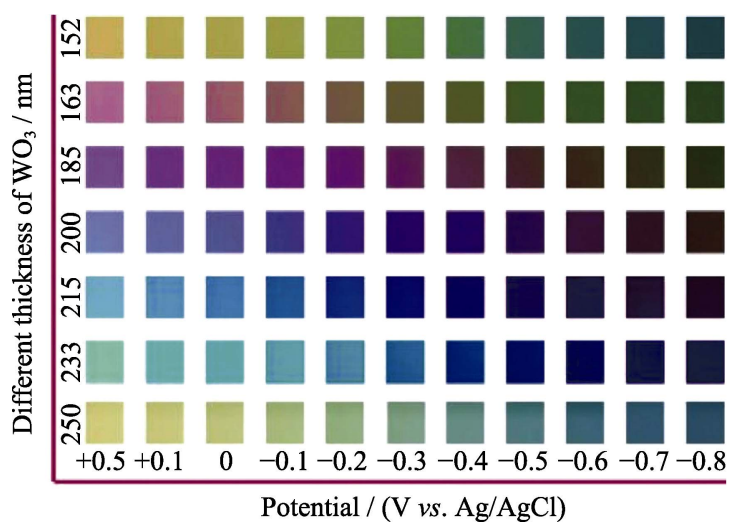

图 3 F-P 纳米谐振腔型电致变色电极在不同外加电位下的 颜色 $^{[24]}$

Fig. 3 Color gallery obtained from F-P nanocavity-type electrochromic electrode at different applied potentials ${ }^{[24]}$ 


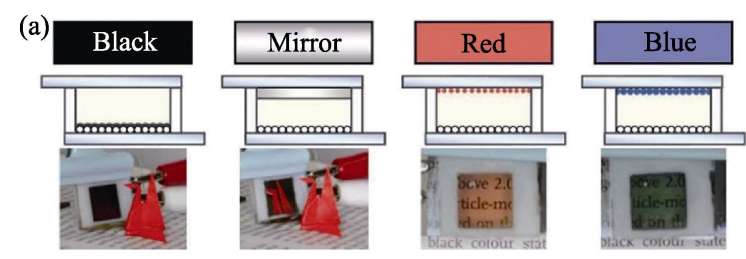

(b)

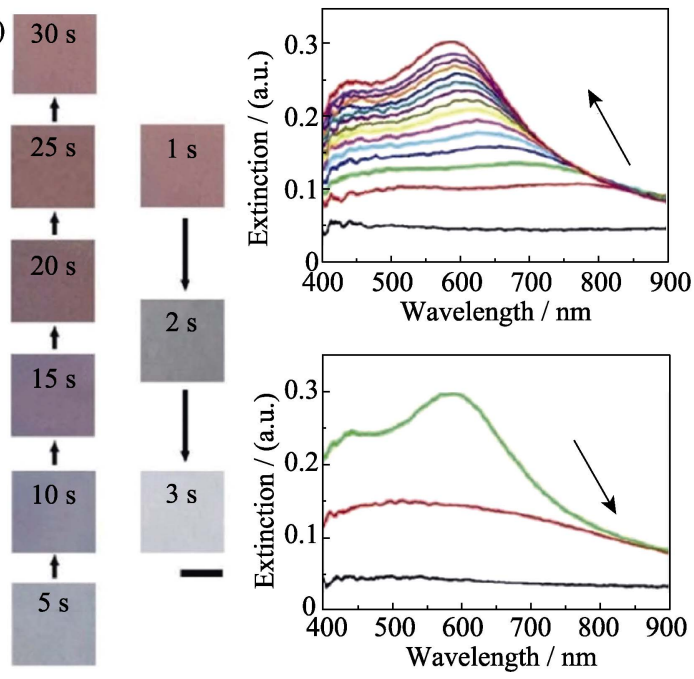

图 4 双电极电致变色电池的结构和照片(a), 在着色(5 30 s) 和褪色过程(1 3 s) 期间的照片和光谱(b) ${ }^{[25-27]}$

Fig. 4 Scheme and photographs of the two-electrode electrochromic cell (a), digital images of the film during coloration (5-30 s) and bleaching process $(1-3 \mathrm{~s})(\mathrm{b})^{[25-27]}$

材料与器件虽然颜色丰富度不如有机材料, 但是通 过合理的设计, 也可以获得较为鲜艳的颜色对比, 在显示、伪装领域展示出较好应用潜力。

\section{2 无机电致变色材料在中远红外波段 的反射特性研究}

中远红外波段即 2 50 $\mu \mathrm{m}$ 的热辐射波段, 探索 材料在中远红外的调控, 可使其应用于红外隐身和 航天器智能热控等领域。对不透明材料, 根据基尔 霍夫定律，在热平衡条件下，材料表面的发射率等 于吸收率，即 1-反射率(公式 2):

$$
\varepsilon=A=1-R
$$

这里 $\varepsilon$ 为材料表面的发射率, $A$ 为材料的吸收率, $R$ 为材料的反射率。

传统固定发射率热控涂层效率低，质量大。 NASA 报告指出 ${ }^{[28]}$, 使用智能热控涂层可以减少热 控质量 $75 \%$, 降低电加热功率 $90 \%$, 在未来航天器 热控涂层技术领域具有较好的应用潜力。

常见的红外波段可调电致变色材料分为有机类 和无机类两种。有机类红外电致变色材料通常采用 导电聚合物作为功能层, 常见的有聚苯胺 ${ }^{[29]}$ (PANI)
和聚噻吩 ${ }^{[30]}$ (PEDOT)。美国早在 2002 年就开始开发 基于 PANI 的红外辐射率可调器件 ${ }^{[15]}$ 。国内李圭课 题组 ${ }^{[11}$ 系统研究了聚苯胺红外调控的原理, 主要是 PANI 掺杂后的导电性变化所致, 他们认为 PANI 膜 层中的极化子和双极化子是 PANI 可以进行红外调 控的根本原因。有机类红外电致变色材料具有柔性 好、可剪裁等优势，但是稳定性较差。无机类红外 电致变色材料通常选用具有半导体性质的材料作为 主要功能层, 最常见的就是氧化铇 $\left(\mathrm{WO}_{3}\right)$ 。相比于有 机类红外电致变色材料, 无机类材料具有更好的稳 定性和耐温性等，应用潜力更大。

\section{$2.1 \mathrm{WO}_{3}$ 的红外调控原理}

在红外波段, $\mathrm{WO}_{3}$ 电致变色的调控受多个因素 共同控制。首先是德鲁德自由电子气体理论, 它主 要针对晶态 $\mathrm{WO}_{3}\left(\mathrm{c}-\mathrm{WO}_{3}\right)$ 。 $\mathrm{c}-\mathrm{WO}_{3}$ 是一种具有高红 外光透过率的半导体, 当 $\mathrm{WO}_{3}$ 变色时, 离子和电子 注入 $\mathrm{WO}_{3}$ 中形成铇青铜。电子在铇离子中进入 $5 \mathrm{~d}$ 轨道, 可以看作是自由电子气体。同时, 离子注入保 持电中性，即 $\mathrm{WO}_{3}$ 具有半导体到金属的过渡态性质， 呈高红外反射特征, 而非晶态 $\mathrm{WO}_{3}\left(\mathrm{a}-\mathrm{WO}_{3}\right)$ 不具有 此特征。反应方程式如下:

$\mathrm{c}-\mathrm{WO}_{3}$ (Fading, semiconductor state) $+x \mathrm{M}^{+}+x \mathrm{e}^{-} \rightleftharpoons$ c- $\mathrm{M}_{x} \mathrm{WO}_{3}$ (Colored, metallic state)

其中, $\mathrm{M}^{+}$代表 $\mathrm{H}^{+} 、 \mathrm{Li}^{+}$等离子。反射率主要基于低频 区 Drude 自由电子气体理论，近似 Hagen-Rubens 关 系 ${ }^{[32]}$, 其表达式如下:

$$
R(\omega)=1-(2 \omega / \pi \sigma)^{1 / 2}
$$

其中, $R(\omega)$ 为角频率的反射率, $\sigma$ 为电子电导率。由 公式可见，随着材料导电性的增加，其红外波段 的反射率也会随之增加。对于 $\mathrm{c}-\mathrm{WO}_{3}, \mathrm{H}^{+}$或者 $\mathrm{Li}^{+}$ 离子注入后, $\mathrm{WO}_{3}$ 的导电性增强, 导致红外反射率 增大。

同时材料中的自由载流子在能带内各个能级之 间的跃迁也可吸收红外光。因此材料导电性是对红 外响应的一个关键因素, 它主要控制了材料对红外 的反射和吸收。

另外, $\mathrm{WO}_{3}$ 着色时的分子振动吸收也会影响红 外发射率。其原理是器件在褪色状态下其变色层对 红外没有吸收, 器件由于高反射红外基底而处于低 红外发射率状态; 而在着色状态下, 由于分子振动 吸收而处于高吸收高发射率状态。

由此可见, 调控 $\mathrm{WO}_{3}$ 或其他电致变色材料在红 外波段的变色特性受多个因素的协同调控作用影响, 而最终调控幅度大小与器件结构设计密切相关。 


\section{2 基于 $\mathrm{WO}_{3}$ 的红外可调电致变色器件设计}

\section{与制备方法}

基于德鲁德自由电子气体原理, Hale 等 ${ }^{[33]}$ 采用 磁控溅射法，分别溅射金 $(\mathrm{Au}) 、 \mathrm{NiO} 、 \mathrm{Ta}_{2} \mathrm{O}_{5} 、 \mathrm{c}-\mathrm{WO}_{3}$ 和 ITO 单层薄膜, 采用中远红外椭偏仪测试各层材 料在 $2 \sim 13.8 \mu \mathrm{m}$ 的折射率 $(n)$ 和消光系数 $(k)$, 根据 $n$ 、 $k$ 值和不同变色层的厚度, 以 $\mathrm{H}^{+}$为变色离子, 模拟 计算了该结构器件的红外发射率。模拟结果表明, $\mathrm{Au} / \mathrm{NiO} / \mathrm{Ta}_{2} \mathrm{O}_{5} / \mathrm{c}-\mathrm{WO}_{3} / \mathrm{ITO}$ 结构的发射率最大变化为 0.538。在实际制备中, 采用与此类似的结构, 依次 溅射铝 $(\mathrm{Al}) 、 \mathrm{a}-\mathrm{WO}_{3} 、 \mathrm{Ta}_{2} \mathrm{O}_{5} 、 \mathrm{c}-\mathrm{WO}_{3}$ 和 $\mathrm{Al}$ 栅极的电 致变色结构, 用 $\mathrm{Li}^{+}$作为变色离子, 用 $\mathrm{a}-\mathrm{WO}_{3}$ 作为电 致变色的互补层, 简化了制备工艺, 得到了发射率 变化为 0.19 的电致变色器件 ${ }^{[34]}$ 。作者还讨论了不同 栅电极结构对器件发射率的影响, 结果表明, 底电 极的红外高反射特性对器件的发射率调节有很大影 响。无论是模拟计算还是实验数据验证, 以下电极为 面电极、上电极为栅格电极的器件比以上电极和下 电极为栅格电极的器件具有更大的调节幅度。

Bessiere 等 ${ }^{[35]}$ 采用水合三氧化铇 $\left(\mathrm{WO}_{3} \cdot \mathrm{H}_{2} \mathrm{O}\right)$ 覆 盖多孔塑料基体, 采用 $\mathrm{Al}$ 栅格电极、钴酸锂 $\left(\mathrm{LiCoO}_{2}\right)$ 作为电解质, 采用层压法制备红外可调器件, 如图 5 所示。由于没有采用真空镀膜法和热处理的方法, 大大简化了器件制备工艺。 $\mathrm{WO}_{3} \cdot \mathrm{H}_{2} \mathrm{O}$ 的晶格结构疏 松, 锂在其中的扩散效率远高于致密结构 $\mathrm{WO}_{3}$ 。利 用该方法, 得到了性能优良的红外发射率可调器件, 发射率变化范围达到 0.3 。从以上研究可以看到, 基 于德鲁德自由电子气体原理设计制备的红外发射率 可调器件的发射率调控幅度均较小, 一个主要的原 因是当材料导电性提升时, 红外反射率提升, 而红 外吸收率也会随之提升, 这在一定程度影响了红外 可调器件的调控幅度。

基于着色褪色时的分子振动吸收理论, Rougier

(a)

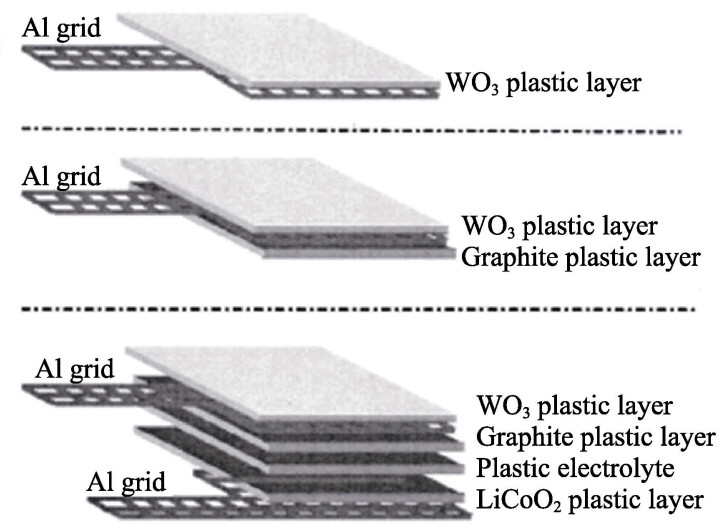

等 ${ }^{\left[{ }^{[6-37]}\right.}$ 以两个半器件结构组装成一个完整的固态器 件, 器件结构为 $\mathrm{Ba}_{2} \mathrm{~F} / \mathrm{Au} / \mathrm{WO}_{3} / \mathrm{Ta}_{2} \mathrm{O}_{5} / \mathrm{NiO} / \mathrm{Au}$, 并对 该器件结构性能进行了模拟计算和实验测试, 实验 结果表明该红外器件在中红外波段和远红外波段的 发射率调控分别达到了 0.27 和 0.18 。

Kislov 等 ${ }^{[38]}$ 以 $\mathrm{WO}_{3}$ 和 $\mathrm{V}_{2} \mathrm{O}_{5}$ 为电致变色材料, 分别在硅 $(\mathrm{Si})$ 和聚酰亚胺 $(\mathrm{PI})$ 祄底上制备了红外可 调的电致变色器件, 红外发射率调控幅度分别为 0.31 和 0.24 。他们还讨论了红外抗反射层的作用, 合 适的红外抗反射层不仅可以保护活性层不受破坏, 而且可以起到减小红外反射、增加调控幅度的作用。 Kislov 等 ${ }^{[39]}$ 还通过实验验证了单纯增加 $\mathrm{WO}_{3}$ 层的 厚度并不能提高器件的红外调控性能, 它主要受粒 子扩散时间和电阻的限制。通过模拟计算, 在最佳 条件下的发射率调控幅度为 0.5 。 Demiryont 等 $^{[40]}$ 以 $\mathrm{WO}_{3}$ 和 $\mathrm{V}_{2} \mathrm{O}_{5}$ 作为电致变色材料, $\mathrm{Li}_{3} \mathrm{~N}$ 和 $\mathrm{F}$ 掺杂 $\mathrm{Li}_{3} \mathrm{~N}$ 作为电解质层制备全固态红外可调电致变色 器件, 并通过红外热像仪比较发射率的变化。其中, 低发射率状态在红外热像仪中显示为蓝色, 高发射 率状态显示为红色，具有明显的抑制或促进红外辐 射能量的效果。热控涂层的面密度仅为 $5 \mathrm{~g} / \mathrm{m}^{2}$, 功 耗仅为 $0.1 \mathrm{~mW} / \mathrm{cm}^{2}$, 发射率变化较大, 7 12 $\mu \mathrm{m}$ 处 平均为 0.8 , 最大为 0.93 , 但是响应时间较慢, 从几 分钟到半小时, 如图 6 所示。

基于上述两种电致变色红外调控机理，均可得 到具有一定红外控制能力的电致变色器件。然而, 中国科学院上海硅酸盐研究所章俞之研究员 ${ }^{[41]}$ 对 ITO 上的 $\mathrm{c}-\mathrm{WO}_{3}$ 着色褪色时发现, 着色态 $\mathrm{c}-\mathrm{WO}_{3}$ 的 红外反射率低于裉色态, 这说明着色后的 $\mathrm{c}-\mathrm{WO}_{3}$ 具 有较高的发射率。同时 Larsson 等 ${ }^{[42]}{ }$ 研究发现, 器 件的红外调节性能可能与其他因素有关。如图 7 所 示，Larsson 制备了一种以 ITO 为祄底、镍钒氧氢 $\left(\mathrm{NiV}_{x} \mathrm{O}_{y} \mathrm{H}_{z}\right)$ 薄膜为离子存储层、 $\mathrm{ZrO}_{2}$ 为离子传导层、

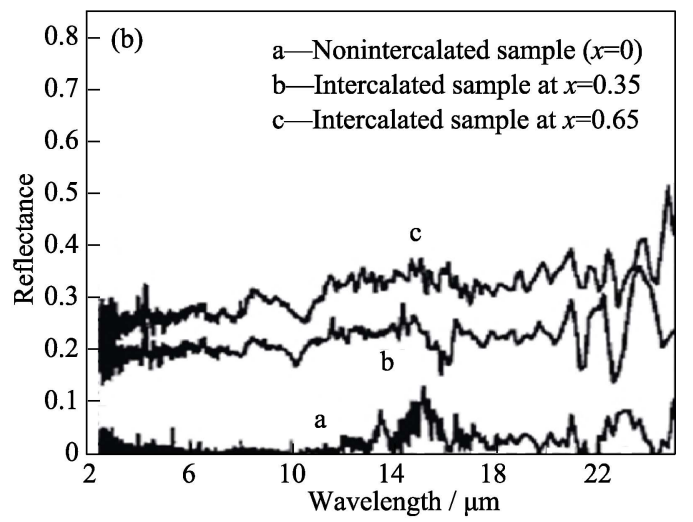

图 5 器件结构示意图(a)和红外反射谱(b) ${ }^{[35]}$

Fig. 5 Schematic diagram of ECD structure (a) and infrared reflectance spectra (b) ${ }^{[35]}$ 

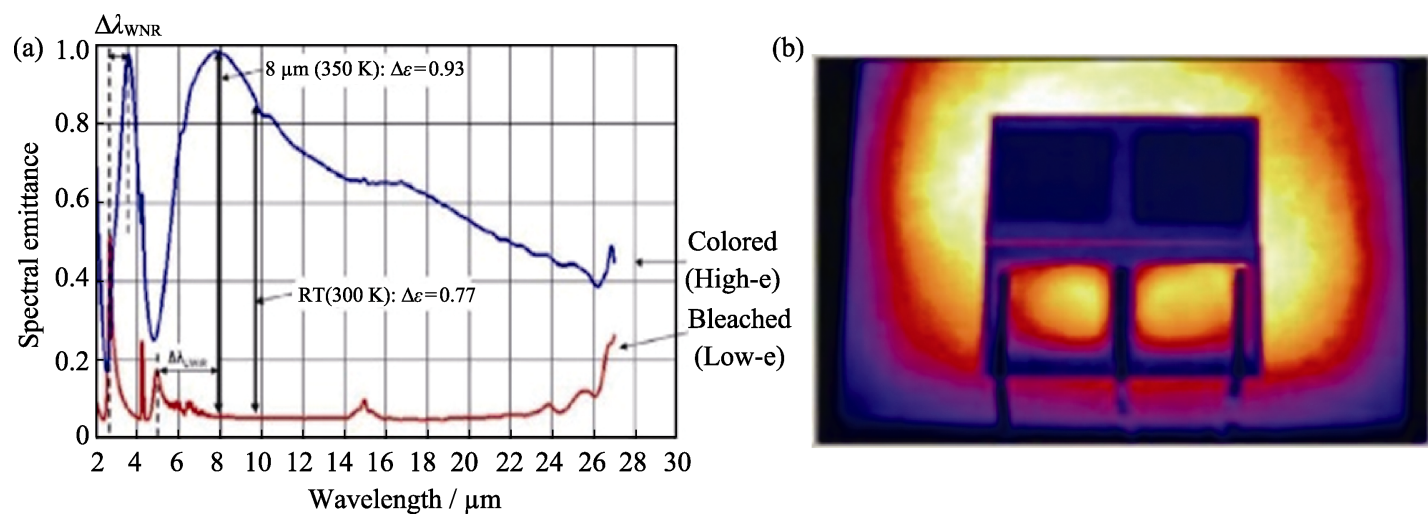

图 6 器件红外光谱发射率 (a) 和红外光学照片 $(b)^{[40]}$

Fig. 6 Spectral emittance of ECD (a) and infrared optical photograph (b) ${ }^{[40]}$
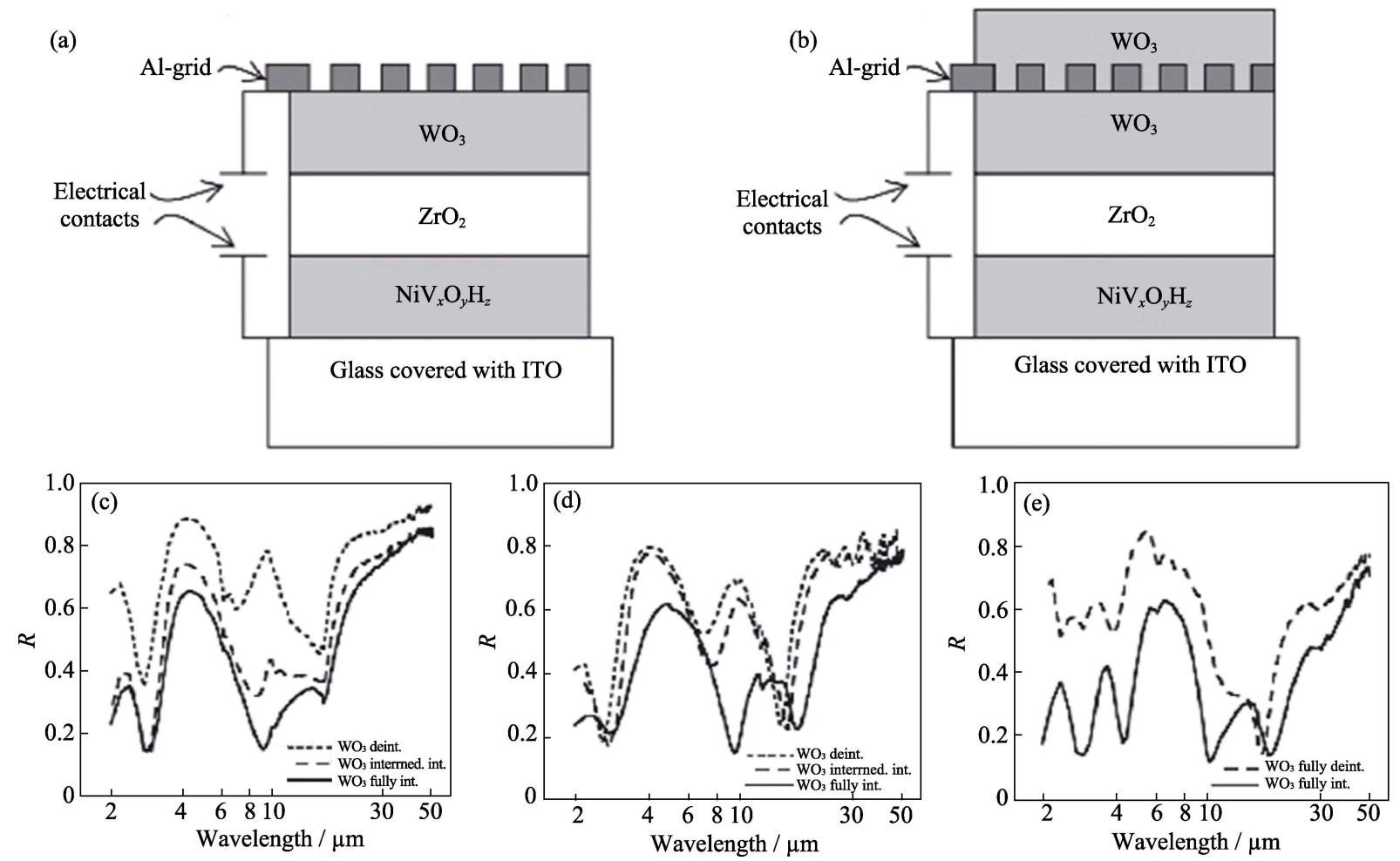

图 7 器件结构示意图 $(a, b)$ 和不同结晶性的氧化铇的红外反射谱 $(c \sim e)^{[42]}$

Fig. 7 Schematic diagrams of ECD structures $(a, b)$ and infrared reflectance spectra with different crystalline $\mathrm{WO}_{3}(\mathrm{c}-\mathrm{e})^{[42]}$

$\mathrm{a}-\mathrm{WO}_{3}$ 和 $\mathrm{c}-\mathrm{WO}_{3}$ 为电致变色红外调控层、 $\mathrm{Al}$ 栅格为 顶电极层的全固态红外调控器件。测试器件的红外 发射率结果表明, 离子注入 $\mathrm{WO}_{3}$ 后, 非晶态和晶态 $\mathrm{WO}_{3}$ 器件的发射率均有所提高。这与其他研究人员 的研究结果不一致, 说明即使同为晶态 $\mathrm{WO}_{3}$ 结构, 红外调控能力变化趋势也不相同。器件的最终红外 性能可能与器件本身的结构设计有关。

哈尔滨工业大学李圭课题组 ${ }^{[43]}$ 综合分析全固 态器件在红外波段变色的影响因素, 避免热处理对 其他膜层的影响, 通过控制溅射功率, 获得了非晶 和晶态 $\mathrm{WO}_{3}$ 层的全固态器件, 分析两者红外发射率 不同的变化趋势, 发现固态器件的红外发射率变化 与 $\mathrm{WO}_{3}$ 的导电性相关, 受德鲁德自由电子气体理论
和红外振动吸收共同控制, 其中 $\mathrm{a}-\mathrm{WO}_{3}$ 器件的调控 幅度更大，达到 0.3 , 如图 8 所示。

综上所述，使用非晶 $\mathrm{WO}_{3}$ 调控红外发射率比多 晶 $\mathrm{WO}_{3}$ 的调控范围大，因为对多晶 $\mathrm{WO}_{3}$ 来说，从绝 缘态着色到半导体态，其导电性的提升是有限的， 因此发射率调节幅度也非常有限。而非晶 $\mathrm{WO}_{3}$ 很容 易从红外完全透明态着色为红外高吸收态，即红外 高发射态，因此具有较大的红外调控幅度。

\section{3 其他红外波段可调无机材料与器件}

$\mathrm{WO}_{3}$ 是最常见的无机红外发射率可调材料，除 $\mathrm{WO}_{3}$ 外, 近年来开发了一些其他红外可调的无机材 料。土耳其 Kocabas 等 ${ }^{[44]}$ 设计了多层石墨烯为红外 调控材料的器件, 在外加电场作用下, 离子嵌入 

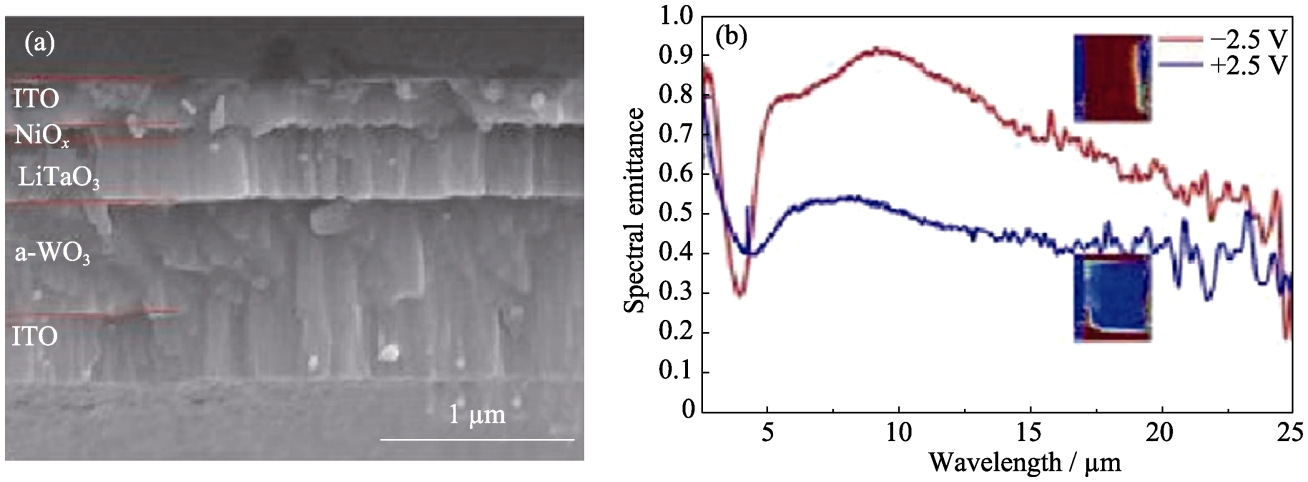

图 8 器件横截面 SEM 照片(a)和器件红外发射率光谱 $(b)^{[43]}$

Fig. 8 Cross-sectional SEM image of the ECD (a) and visible-to-infrared spectral emittance (b) ${ }^{[43]}$

多层石墨烯中，石墨烯的费米能级发生变化，表面 高迁移率载流子浓度增加，导电性增大，表面方阻 下降，辐射能力减弱，如图 9, 对不同厚度石墨烯的 红外调控能力进行研究, 获得的最大红外发射率变 化幅度为 0.5 。

美国 Yang 等 ${ }^{[45]}$ 设计了基于 $\mathrm{Li}_{4} \mathrm{Ti}_{5} \mathrm{O}_{12}$ (LTO)的红

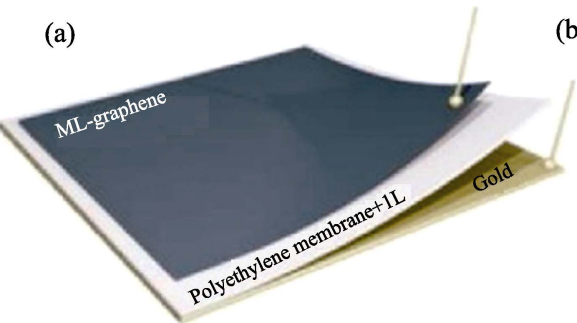

(c)

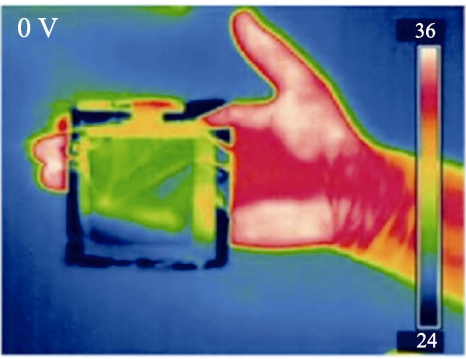

外调控器件, 与石墨烯类似, 当 Li 插层 LTO 后, LTO 费米能级发生变化, LTO 从宽禁带半导体态转 变为金属态，导电性上升。与石墨烯结果不同的是， 由于吸收自由载流子，材料的红外发射率有明显提 升, 最优厚度的 LTO 红外调控幅度达到 0.3 , 如图 10 所示。

(b) Thermal radiation

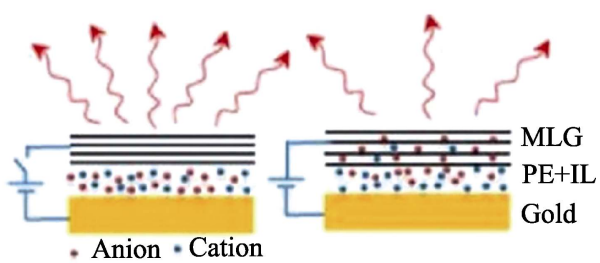

(d)

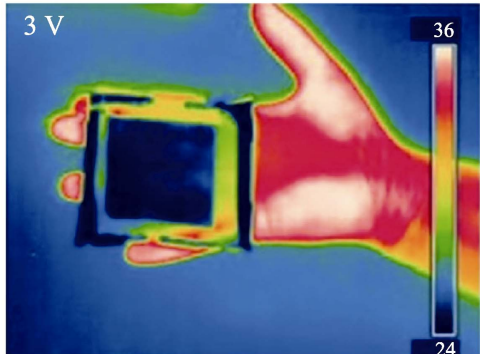

图 9 石墨烯器件结构示意图(a), 石墨烯器件工作原理示意图(b), 0 和 $3 \mathrm{~V}$ 电压下的热相机图像 $(\mathrm{c}, \mathrm{d})^{[44]}$

Fig. 9 Schematic drawing of graphene device (a), schematic representation of working principle of the graphene device (b), thermal camera images of device under the voltage bias of 0 and $3 \mathrm{~V}$, respectively $(\mathrm{c}, \mathrm{d})^{[44]}$
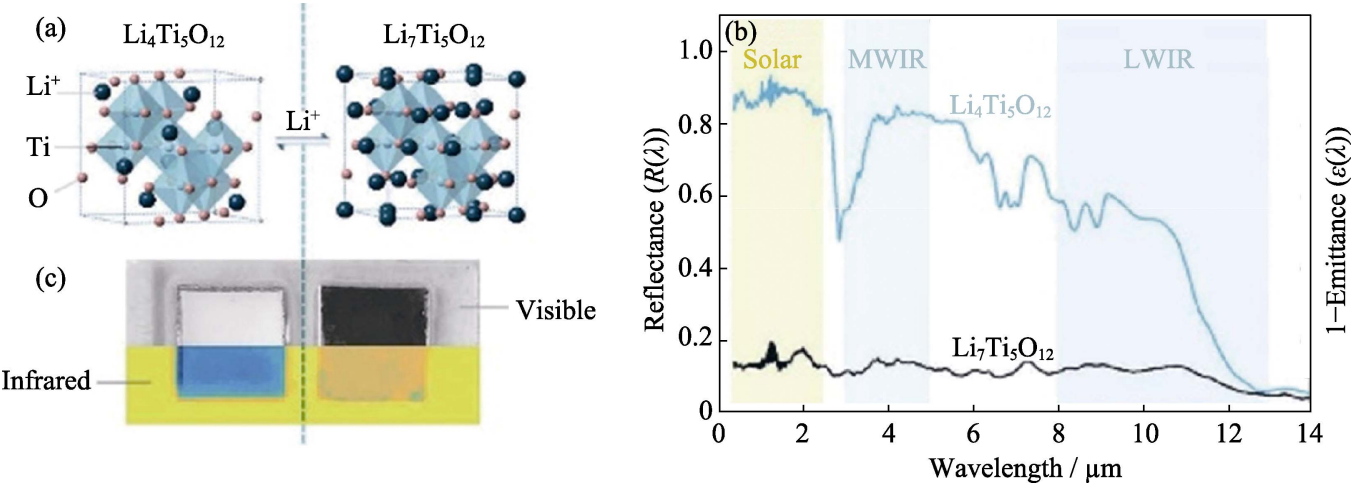

图 10 LTO 晶体结构(a), LTO 反射光谱(b)和 LTO 器件红外调控实物图(c) ${ }^{[45]}$

Fig. 10 Crystal structures of $\mathrm{Li}_{4} \mathrm{Ti}_{5} \mathrm{O}_{12}$ and $\mathrm{Li}_{7} \mathrm{Ti}_{5} \mathrm{O}_{12}$ (a), visible-to-infrared spectral reflectance (b), photograph (top) and thermograph (bottom) in the two states (c) ${ }^{[45]}$ 


\section{3 结束语}

电致变色是近年来的研究热点, 无机变色材料 性能稳定, 耐候性好, 最具应用潜力。目前基于透过 性能的无机电致变色材料研究较为广泛成熟, 基于 反射性能的无机变色材料也受到了较大关注。在可 见波段, 通过对 $\mathrm{V}_{2} \mathrm{O}_{5}$ 掺杂、颜色复配、构建一维光 子晶体、法布里一珀罗纳米谐振腔、贵金属纳米粒子 微纳结构等方式, 开发了多种具有鲜艳颜色转变的 无机电致变色材料与器件。在红外波段依据不同的 调控原理, 设计开发了多种红外发射率可调的器 件。总体来看, 从可见到中远红外波段, 对于无机变 色材料的反射特性研究仍处于基本的原理探索和原 型器件的研发上, 因此还没得到广泛的应用。增加 无机材料颜色的丰富度和器件的优化整合是未来无 机反射型电致变色材料与器件的发展方向, 结合无 机材料高稳定型的固有特性, 使无机反射型电致变 色材料具有更广阔的应用前景。

\section{参考文献:}

[1] DEB S. A novel electrophotographic system. Applied Optics, 1969, 8(101): 192-195.

[2] ZHANG X, LI W J, LI Y, et al. Research progress of inorganic all-solid-state electrochromic devices. Materials Science and Technology, 2020, 28: 140-149.

[3] CHEN X, DOU S L, LI W J, et al. All solid state electrochromic devices based on the LiF electrolyte. Chemical Communications, 2020, 56: 5018-5021.

[4] LI W J, ZHANG X, CHEN X, et al. Effect of independently controllable electrolyte ion content on the performance of all-solid-state electrochromic devices. Chemical Engineering Journal, 2020, 398: 125628.

[5] GREER B. Control System for Electrochromic Devices. U.S. Patent 7277215. 2007-10-2.

[6] WANG Z, PRADHAN A, ROZBICKI R. Electrochromic Devices. U.S. Patent 8764951. 2014-7-1.

[7] XU Q F, LI J, ZHAO J. A Kind of Electrochromic Glass. China, CN204595399U. 2015-8-26.

[8] ZHAO Y M, ZHANG X, CHEN X, et al. Preparation of $\mathrm{WO}_{3}$ films with controllable crystallinity for improved near-Infrared electrochromic performances. ACS Sustainable Chemistry \& Engineering, 2020, 8(31): 11658-11666.

[9] XIA X H, TU J P, ZHANG J, et al. Morphology effect on the electrochromic and electrochemical performances of $\mathrm{NiO}$ thin films. Electrochimica Acta, 2008, 53(18): 5721-5724.

[10] LI W J, ZHANG X, CHEN X, et al. Lithiation of $\mathrm{WO}_{3}$ films by evaporation method for all-solid-state electrochromic devices. Electrochimica Acta, 2020, 355: 136817.

[11] ZHOU D, XIE D, XIA X H, et al. All-solid-state electrochromic devices based on $\mathrm{WO}_{3} \mid \mathrm{NiO}$ films: material developments and future applications. Science China Chemistry, 2017, 60(1): 3-12.

[12] SHENG M F, ZHANG L P, WEST L J, et al. Multicolor electrochromic dye-doped liquid crystal yolk-shell microcapsules. ACS Applied Materials \& Interfaces, 2020, 12(26): 29728-29736.
[13] MORTIMER R J, DYER A L, REYNOLDS J R. Electrochromic organic and polymeric materials for display applications. Displays, 2006, 27: 2-18.

[14] YU H T, SHAO S, YAN L J, et al. Side-chain engineering of green color electrochromic polymer materials: toward adaptive camouflage application. Journal of Materials Chemistry C, 2016, 4: 2269-2273.

[15] CHANDRASEKHAR P, ZAY B J, BIRUR G C, et al. Large, switchable electrochromism in the visible through far-infrared in conducting polymer devices. Advanced Functional Materials, 2002, 12(2): 95-103.

[16] CHERNOVA N A, ROPPOLO M, DILLON A C, et al. Layered vanadium and molybdenum oxides: batteries and electrochromics. Journal of Materials Chemistry, 2009, 19: 2526-2552.

[17] TONG Z Q, LI N, LV H M, et al. Annealing synthesis of coralline $\mathrm{V}_{2} \mathrm{O}_{5}$ nanorod architecture for multicolor energy-efficient electrochromic device. Solar Energy Materials and Solar Cells, 2016, 146: 135-143.

[18] ZHAO G F, WANG W Q, WANG X L, et al. A multicolor electrochromic film based on a $\mathrm{SnO}_{2} / \mathrm{V}_{2} \mathrm{O}_{5}$ core/shell structure for adaptive camouflage. Journal of Materials Chemistry C, 2019, 7: 5702-5709.

[19] ZHANG X, LI W J, CHEN X, et al. Inorganic all-solid-state electrochromic devices with reversible color change between yellow-green and emerald green. Chemical Communications, 2020, 56: 10062-10065.

[20] ZHANG W, LI H Z, YU W W, et al. Transparent inorganic multicolour displays enabled by zinc-based electrochromic devices. Light: Science \& Applications, 2020, 9(1): 121.

[21] BLANCO A, CHOMSKI E, GRABTCHAK S, et al. Large-scale synthesis of a silicon photonic crystal with a complete three-dimensional bandgap near 1.5 micrometres. Nature, 2000, 405(6785): $437-440$

[22] REDEL E, MLYNARSKI J, MOIR J, et al. Electrochromic Bragg mirror: ECBM. Advanced Materials, 2012, 24(35): 265-269.

[23] XIAO L L, LV Y, LIN J, et al. $\mathrm{WO}_{3}$-based electrochromic distributed Bragg reflector: toward electrically tunable microcavity luminescent device. Advanced Optical Materials, 2018, 6(1): 1-8.

[24] WANG Z, WANG X Y, CONG S, et al. Towards full-colourtunability of inorganic electrochromic devices using ultracompact Fabry-Perot nanocavities. Nature Communications, 2020, 11(1): 1-9.

[25] ARAKI S, NAKAMURA K, KOBAYASHI K, et al. Electrochemical optical-modulation device with reversible transformation between transparent, mirror, and black. Advanced Materials, 2012, 24(23): 122-126.

[26] TSUBOI A, NAKAMURA K, KOBAYASHI N, et al. A localized surface plasmon resonance-based multicolor electrochromic device with electrochemically size-controlled silver nanoparticles. Advanced Materials, 2013, 25(23): 3197-3201

[27] LI N, WEI P, YU L, et al. Dynamically switchable multicolor electrochromic films. Small, 2019, 15(7): 1-7.

[28] SWANSON T D, BIRUR G C. NASA thermal control technologies for robotic spacecraft. Applied Thermal Engineering, 2003, 23(9): 1055-1065.

[29] LI H, XIE K, PAN Y, et al. Variable emissivity infrared electrochromic device based on polyaniline conducting polymer. Synthetic Metals, 2009, 159(13): 1386-1388.

[30] LOUET C, CANTIN S, DUDON J P, et al. A comprehensive study of infrared reflectivity of poly (3, 4-ethylenedioxythiophene) model layers with different morphologies and conductivities. Solar 
Energy Materials and Solar Cells, 2015, 143: 141-151.

[31] ZHANG L P, WANG B, LI X B, et al. Further understanding of the mechanisms of electrochromic devices with variable infrared emissivity based on polyaniline conducting polymers. Journal of Materials Chemistry C, 2019, 7(32): 9878-9891.

[32] MODINE F A, SMITH D Y. Approximate formulas for the amplitude and the phase of the infrared reflectance of a conductor. Journal of The Optical Society of America A-Optics Image Science and Vision, 1984, 1(12): 1171-1174.

[33] HALE J S, WOOLlam J A. Prospects for IR emissivity control using electrochromic structures. Thin Solid Films, 1999, 339(1): 174-180.

[34] FRANKE E B, TRIMBLE $\mathrm{C}$ L, SCHUBERT $\mathrm{M}$, et al. All-solid-state electrochromic reflectance device for emittance modulation in the far-infrared spectral region. Applied Physics Letters, 2000, 77(7): 930-932.

[35] BESSIERE A, MARCEL C, MORCRETTE M, et al. Flexible electrochromic reflectance device based on tungsten oxide for infrared emissivity control. Journal of Applied Physics, 2002, 91(3): 1589-1594.

[36] SAUVET K, SAUQUES L, ROUGIER A, et al. IR electrochromic $\mathrm{WO}_{3}$ thin films: from optimization to devices. Solar Energy Mater. Solar Cells, 2009, 93: 2045-2049.

[37] SAUVET K, SAUQUES L, ROUGIER A, et al. Electrochromic properties of $\mathrm{WO}_{3}$ as a single layer and in a full device: from the visible to the infrared. Journal of Physics and Chemistry of Solids, 2010, 71: 696-699.
[38] KISLOV N, GROGER H, PONNAPPAN R. All-solid-state electrochromic variable emittance coatings for thermal management in space. AIP Conference Proceedings, 2003, 654(1): $172-179$.

[39] KISLOV N, GROGER H, PONNAPPAN R, et al. Electrochromic variable emittance devices on silicon wafer for spacecraft thermal control. AIP Conference Proceedings, 2004, 699(1): 112-118.

[40] DEMIRYONT H, MOOREHEAD D. Electrochromic emissivity modulator for spacecraft thermal management. Solar Energy Materials and Solar Cells, 2009, 93(12): 2075-2078.

[41] HUANG Y S, ZHANG Y Z, ZENG X T, et al. Study on Raman spectra of electrochromic $\mathrm{c}-\mathrm{WO}_{3}$ films and their infrared emittance modulation characteristics. Applied Surface Science, 2002, 202(1): 104-109.

[42] LARSSON A L, NIKLASSON G A. Infrared emittance modulation of all-thin-film electrochromic devices. Materials Letters, 2004, 58(20): 2517-2520.

[43] ZHANG X, TIAN Y L, LI W J, et al. Preparation and performances of all-solid-state variable infrared emittance devices based on amorphous and crystalline $\mathrm{WO}_{3}$ electrochromic thin films. Solar Energy Materials and Solar Cells, 2019, 200: 109916.

[44] SALIHOGLU O, UZLU H B, YAKAR O, et al. Graphene-based adaptive thermal camouflage. Nano Letters, 2018, 18(7): 4541-4548.

[45] MANDAL J, DU S, DONTIGNY $\mathrm{M}$, et al. $\mathrm{Li}_{4} \mathrm{Ti}_{5} \mathrm{O}_{12}:$ a visible-to-infrared broadband electrochromic material for optical and thermal management. Advanced Functional Materials, 2018, 28(36): $1-8$. 